\title{
Correction to: Serotype distribution of Streptococcus pneumoniae among healthy carriers and clinical patients: a systematic review from Iran
}

\author{
Maryam Alizadeh Chamkhaleh ${ }^{1} \cdot$ Abdoulreza Esteghamati $^{2} \cdot$ Shirin Sayyahfar $^{2} \cdot$ Alireza Gandomi-Mohammadabadi $^{1}$. \\ Javad Balasi ${ }^{1}$ • Hossein Abdiaei ${ }^{1}$ • Yousef Moradi ${ }^{3}$ • Maziar Moradi-Lakeh ${ }^{4,5}$
}

Published online: 29 October 2020

(C) Springer-Verlag GmbH Germany, part of Springer Nature 2020

\section{Correction to: European Journal of Clinical Microbiology \& Infectious Diseases.} https://doi.org/10.1007/s10096-020-03963-z

In the originally published article, the name of the 6th author was incorrectly presented as Hossein Abdiae. The correct name is Hossein Abdiaei, which is also given above.

The original article has been corrected.

Publisher's note Springer Nature remains neutral with regard to jurisdictional claims in published maps and institutional affiliations.

The online version of the original article can be found at https://doi.org/ 10.1007/s10096-020-03963-Z

Abdoulreza Esteghamati esteghamati@gmail.com

1 Student Research Committee, School of Medicine, Iran University of Medical Sciences, Tehran, Iran

2 Research Center of Pediatric Infectious Diseases, Institute of Immunology and Infectious Diseases, Iran University of Medical Sciences, Tehran, Iran

3 Department of Epidemiology, Iran University of Medical Sciences, Tehran, Iran

4 Department of Community Medicine, Iran University of Medical Sciences and Health Services, Tehran, Iran

5 Institute for Health Metrics and Evaluation, University of Washington, Seattle, WA, USA 